\title{
Efficacy and Safety of Nab-Paclitaxel as Second-line Chemotherapy for Locally Advanced and Metastatic Non-small Cell Lung Cancer
}

\author{
WENJING WONG ${ }^{1,2^{*}}$, PING SUN ${ }^{2 *}$, ZHENGBIN MU ${ }^{2}$, JIANNAN LIU ${ }^{2}$, CAIYAN YU $^{2}$ and AINA LIU ${ }^{2}$ \\ ${ }^{1}$ Department of Oncology, Jinan Central Hospital, Affiliated to Shandong University, Jinan, P.R. China; \\ ${ }^{2}$ Department of Oncology, Yuhuangding Hospital of Yantai, Yantai, P.R. China
}

\begin{abstract}
Aim: To investigate the efficacy and safety of nanoparticle albumin-bound paclitaxel (nab-paclitaxel) for locally advanced or metastatic non-small cell lung cancer (NSCLC) as second-line chemotherapy. Patients and Methods: We retrospectively reviewed the treatment of 34 patients with advanced NSCLC whose first-line treatment had failed. These patients received nab-paclitaxel $260 \mathrm{mg} / \mathrm{m}^{2}$ on day 1 and day 8 of a 21-day cycle from July 2014 to February 2016. One cycle of treatment lasted 3 weeks and all patients completed more than two cycles. All patients were assessed for adverse events related to treatment. Results: No patient achieved complete response (CR); 12 patients reached partial response $(P R), 12$ patients achieved stable disease $(S D)$ and 10 patients progressive disease (PD). The overall response rate (ORR) was $35.3 \%$ and the disease control rate (DCR) $70.6 \%$. There was no significant difference in either ORR or DCR within the subgroups. The median progression-free survival (PFS) was 5.7 months (95\% confidence interval $(C I)=3.8-7.6)$ and the median overall survival (OS) was 9 months (95\% CI=8.3-9.7). There was no statistical difference in $O S(p=0.066)$, but subgroup analysis showed that patients with squamous carcinoma benefited more in PFS (the median PFS of squamous carcinoma vs. adenocarcinoma was 7.3 months vs. 5 months, $p=0.001$ ). Major adverse events included myelosuppression, gastrointestinal response, baldness, myalgia and neurotoxicity. Hypersensitivity reactions were not reported. Conclusion: Nab-paclitaxel is an effective chemotherapy for
\end{abstract}

\footnotetext{
*These Authors contributed equally to this study.

Correspondence to: Dr. Aina Liu, Department of Oncology, Yuhuangding Hospital of Yantai, 20 Yudong Road, Zhifu District, Yantai, Shandong Province 264001, P.R. China. Tel: +86 13906388003, e-mail: nana4312@sina.com
}

Key Words: NSCLC, nab-paclitaxel, chemotherapy, second-line. locally advanced and metastatic NSCLC as treatment and has a superior application prospect for squamous NSCLC. Toxicity is generally mild and manageable.

Lung cancer has become one of the leading causes of death worldwide. Most patients diagnosed with non-small cell lung cancer (NSCLC) already have advanced disease, with approximately 1.4 million patients dying of NSCLC every year (1). Recurrence and metastasis usually occur in advanced NSCLC after standard first-line therapy. Secondline therapies, despite fast development, are targeted, generally specific for certain subgroups and, thus, remain expensive. Chemotherapy is still important as second-line treatment for locally advanced and metastatic NSCLC and taxanes, such as paclitaxel and docetaxel, play important roles in the second-line treatment of NSCLC. However, both require solvents, which have been associated with drug delivery issues, hepatic toxicity, hypersensitivity reactions and peripheral neuropathy. These toxic effects may lead to decreased dosage and interruption of chemotherapy, thus reducing efficacy. Therefore, chemotherapy drugs that have greater efficacy and lower toxicity profiles are required for patients with advanced NSCLC.

Nanoparticle albumin-bound paclitaxel (nab-paclitaxel) is a new type of paclitaxel that, compared to conventional solvent-based paclitaxel, has many advantages, such as higher efficiency, lower toxicity and more usage convenience. This is mainly due to the fact that nabpaclitaxel is comprised of albumin and paclitaxel to form nanoparticles devoid of any solvents or ethanol, thus greatly reducing the chances of allergic reactions and toxicity and therefore cancelling the need of any pre-medications given prior to its administration. Based on this background, 34 patients with locally advanced and metastatic NSCLC whose first-line chemotherapy failed were enrolled in this study between July 2014 and February 2016. They were treated with nab-paclitaxel at the Yuhuangding Hospital of Yantai. This study analyzed the clinical efficacy and adverse effects 
Table I. Clinical features of the 34 investigated patients.

\begin{tabular}{lrc}
\hline Clinical features & $\mathrm{N}$ & Percentage \\
\hline Gender & & \\
$\quad$ Male & 26 & 76.47 \\
$\quad$ Female & 8 & 23.53 \\
Age & & \\
$\quad \leq 65$ & 20 & 58.82 \\
$\quad>65$ & 14 & 41.18 \\
ECOG & & \\
$0-1$ & 15 & 44.12 \\
2 & 19 & 55.88 \\
Smoking status & & \\
$\quad$ Smoking & 16 & 47.06 \\
$\quad$ Non-smoking & 18 & 52.94 \\
Pathology & & \\
$\quad$ Adenocarcinoma & 22 & 64.7 \\
$\quad$ Squamous carcinoma & 12 & 35.3 \\
Clinical stage & & \\
$\quad$ Stage III & 5 & 14.7 \\
$\quad$ Stage IV & 29 & 85.3 \\
Previous taxane & & \\
$\quad$ Yes & 14 & 41.18 \\
$\quad$ No & 20 & 58.82 \\
Previous radiotherapy & & \\
$\quad$ Yes & 13 & 61.77 \\
$\quad$ No & 21 & \\
\hline
\end{tabular}

ECOG, Eastern Cooperative Oncology Group.

of nab-paclitaxel, as second-line therapy, in patients with advanced NSCLC.

\section{Materials and Methods}

Entry criteria. (i) Age $\geq 18$ years and patients who had histologically confirmed NSCLC. All tumors of these patients were assessable by computed tomography (CT) scan. (ii) Eastern Cooperative Oncology Group (ECOG) score of 0 2. (iii) Patients who failed their first-line therapy. (iv) Patients who had recovered from the toxic effects of any previous treatment. (v) Normal routine blood tests with liver and kidney functions $\leq 2.5$ and $\leq 1.5$ times of the normal range, respectively. (vi) Life expectancy of more than three months. (vii) Signed consent for chemotherapy before treatment.

Clinical data. According to the entry criteria, 34 cases (26 male and 8 female) of advanced NSCLC were enrolled in this study at the Oncology Department of Yuhuangding Hospital, Yantai, China (Table I). Five cases were in stage III, whilst 29 cases in stage IV. Sixteen patients had a history or smoking and the other 18 were non-smokers. Of the 34 cases, 12 were squamous cancer and 22 cases adenocarcinoma. Fourteen cases had previously received taxanes and 20 cases had not.

Methods. Patients were given nab-paclitaxel at a dose of $260 \mathrm{mg} / \mathrm{m}^{2}$ on day 1 and day 8 of every 21 days. Treatment was repeated every three weeks, unless adverse toxicity or disease progression occurred or if the patient refused to continue treatment. When adverse effects
Table II. Correlation between clinical features and short-term effect of nab-paclitaxel for advanced NSCLC as a second-line chemotherapy.

\begin{tabular}{|c|c|c|c|c|c|c|c|c|}
\hline & CR & PR & SD & PD & ORR & $p$-Value & DCR & $p$-Value \\
\hline \multicolumn{9}{|l|}{ Gender } \\
\hline Male & 0 & 10 & 9 & 7 & 10 & 0.486 & 19 & 0.566 \\
\hline Female & 0 & 2 & 3 & 3 & 2 & & 5 & \\
\hline \multicolumn{9}{|l|}{ Age } \\
\hline$\leq 65$ & 0 & 6 & 8 & 6 & 6 & 0.44 & 14 & 0.928 \\
\hline$>65$ & 0 & 6 & 4 & 4 & 6 & & 10 & \\
\hline \multicolumn{9}{|l|}{ ECOG } \\
\hline $0-1$ & 0 & 4 & 5 & 6 & 4 & 0.35 & 9 & 0.229 \\
\hline 2 & 0 & 8 & 7 & 4 & 8 & & 15 & \\
\hline \multicolumn{9}{|l|}{ Smoking status } \\
\hline Smoking & 0 & 6 & 5 & 5 & 6 & 0.8 & 11 & 0.824 \\
\hline Non-smoking & 0 & 6 & 7 & 5 & 6 & & 13 & \\
\hline \multicolumn{9}{|l|}{ Pathology } \\
\hline Adenocarcinoma & 0 & 10 & 8 & 4 & 10 & 0.093 & 18 & 0.052 \\
\hline Squamous carcinoma & 0 & 2 & 4 & 6 & 2 & & 6 & \\
\hline \multicolumn{9}{|l|}{ Clinical stage } \\
\hline Stage III & 0 & 2 & 3 & 0 & 2 & 0.812 & 5 & 0.118 \\
\hline Stage IV & 0 & 10 & 9 & 10 & 10 & & 19 & \\
\hline \multicolumn{9}{|l|}{ Previous taxane } \\
\hline Yes & 0 & 4 & 5 & 5 & 4 & 0.493 & 9 & 0.5 \\
\hline No & 0 & 8 & 7 & 5 & 8 & & 15 & \\
\hline \multicolumn{9}{|l|}{ Previous radiotherapy } \\
\hline Yes & 0 & 4 & 5 & 4 & 4 & 0.664 & 9 & 0.891 \\
\hline No & 0 & 8 & 7 & 6 & 8 & & 15 & \\
\hline
\end{tabular}

NSCLC, Non-small cell lung cancer; ECOG, Eastern Cooperative Oncology Group; CR, complete response; PR, partial response; SD, stable disease; PD, progressive disease, ORR, overall response rate, $\mathrm{DCR}$, disease control rate.

of nab-paclitaxel appeared, suspension or reduction of the drug dose was permitted. The efficacy was evaluated every two cycles by imaging examinations and recording objective lesions, quality of life and adverse reactions. Hematologic examinations were performed routinely during the treatment with nab-paclitaxel.

Assessment and adverse reactions. (a) Short-term effects: The objective efficacy was evaluated by the Response Evaluation Criteria in Solid Tumors (RECIST) 1.1. The therapeutic evaluation included complete response (CR), partial response (PR), stable disease (SD) and progressive disease (PD). CR plus PR represented overall response rate (ORR), CR plus $\mathrm{PR}$ and $\mathrm{SD}$ represented disease control rate (DCR).

(b) Long-term effects: progression-free survival (PFS) was defined as the time from the first use of nab-paclitaxel to progression of disease. Overall survival (OS) was defined as the time from the first use of the drug to death or the date of the last visit.

(c) The evaluation of adverse events relied on the NCI Common Toxicity Criteria (CTC) Version 4.0.

Follow-up. The follow-up time was from the time patients enrolled in the study to December 31, 2016.

Statistical analysis. SPSS 22 software (IBM, Armonk, NY, USA) was used for statistical analysis. Correlation between clinical characteristics and effects was analyzed using a $\chi^{2}$ test. 


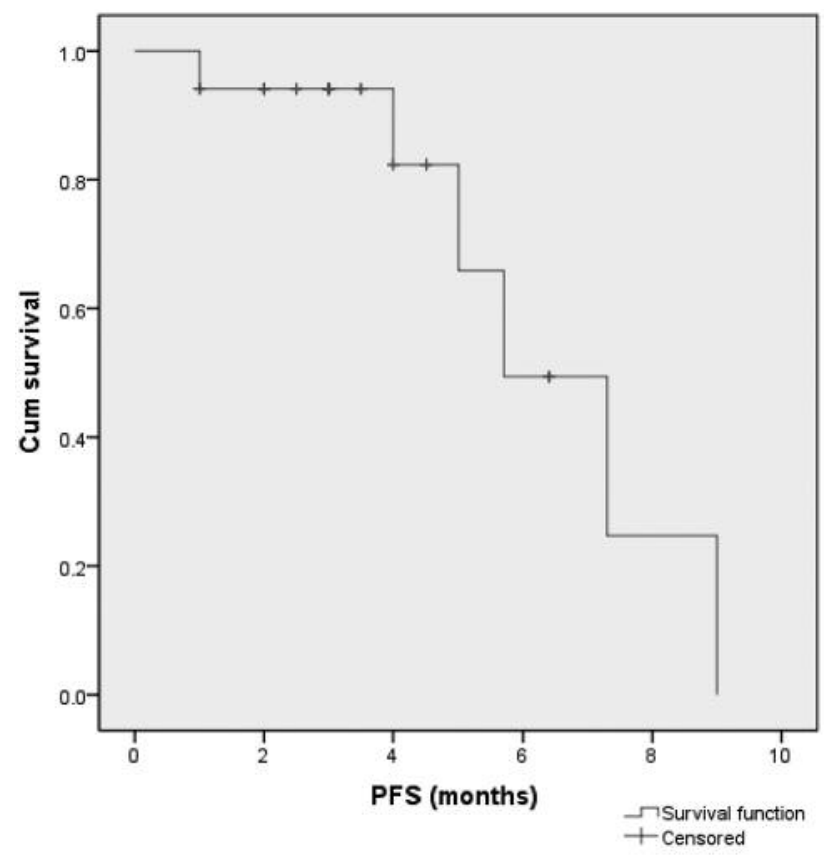

Figure 1. Progression-free survival (PFS) of 34 patients who received nab-paclitaxel as second-line chemotherapy. The median PFS was 5.7 months $(95 \%$ confidence interval $(C I)=3.8-7.6)$.

Kaplan-Meier was used to draw the survival curve. A value of $p<0.05$ was considered to be statistically significant.

\section{Results}

Short-term effects. Thirty-four patients completed a total of 154 cycles of chemotherapy; the median chemotherapy cycles completed were 4 . All patients were evaluated with outcomes of which no patient had CR; 12 patients had PR (35.3), 12 patients had SD (35.3\%), 10 patients had PD (29.4\%). The ORR was $35.3 \%$, while the DCR was $70.6 \%$. According to the analysis, there was no significant difference in subgroups in either ORR or DCR. Table II shows correlation between clinical features and short-term effect of nab-paclitaxel as second-line chemotherapy for advanced NSCLC.

Long-term effects. The median PFS was 5.7 months (95\% confidence interval $(\mathrm{CI})=3.8-7.6$; Figure 1$)$ and the median $\mathrm{OS}$ was 9 months (95\% CI=8.3-9.7; Figure 2). Subgroup analysis showed that patients with squamous lung carcinoma benefited more in PFS compared to patients with adenocarcinoma (the median PFS of squamous carcinoma $v s$. adenocarcinoma was 7.3 months $v s .5$ months, $p=0.001$; Figure 3 ). In contrast, no similar result was found in OS $(p=0.066)$.

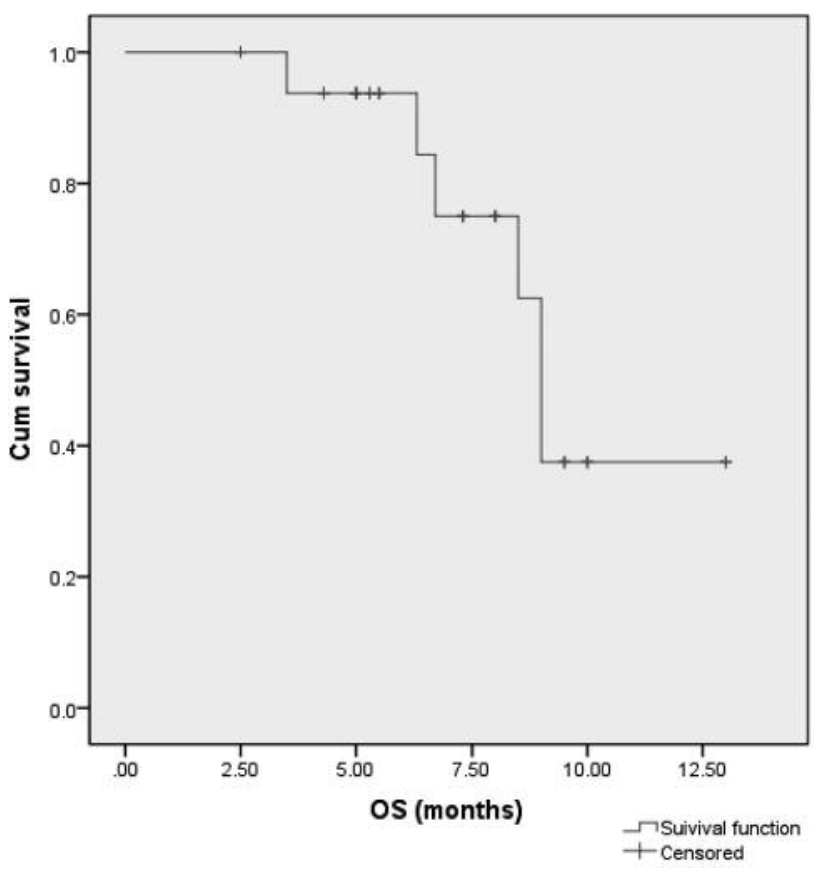

Figure 2. Overall survival (OS) of 34 patients who received nabpaclitaxel as second-line chemotherapy. The median OS was 9 months (95\% confidence interval $(C I)=8.3-9.7)$.

Adverse drug reaction. According to our review, treatmentrelated toxicities were tolerable. The most common toxicity was hematological toxicity, including neutropenia (52.9\%) and thrombocytopenia (23.5\%). Other adverse effects were peripheral neuropathy $(14.7 \%)$, gastrointestinal response (20.6\%), myalgia (17.6\%) and baldness (20.6\%). This was similar with phase III trials of nab-paclitaxel combination regimens in patients with unselected advanced NSCLC(2). Four cases $(11.76 \%)$ had grade III/IV adverse reactions. No febrile neutropenia was observed. Most of these adverse events, except baldness, were easily managed. No serious adverse event or hypersensitivity occurred during the study. Table III shows the adverse effects of nab-paclitaxel experienced by the 34 patients.

\section{Discussion}

The incidence and mortality of lung cancer is high in China, with $75-80 \%$ of the patients being diagnosed as NSCLC (3). Most of these are inoperable due to the advanced disease stage at time of diagnosis. Advanced lung cancer is prone to recurrence and metastasis and most patients receive treatment beyond first-line recommendation.

Paclitaxel is widely used for the treatment of NSCLC as second-line chemotherapy; however, this treatment is commonly associated with adverse events. 


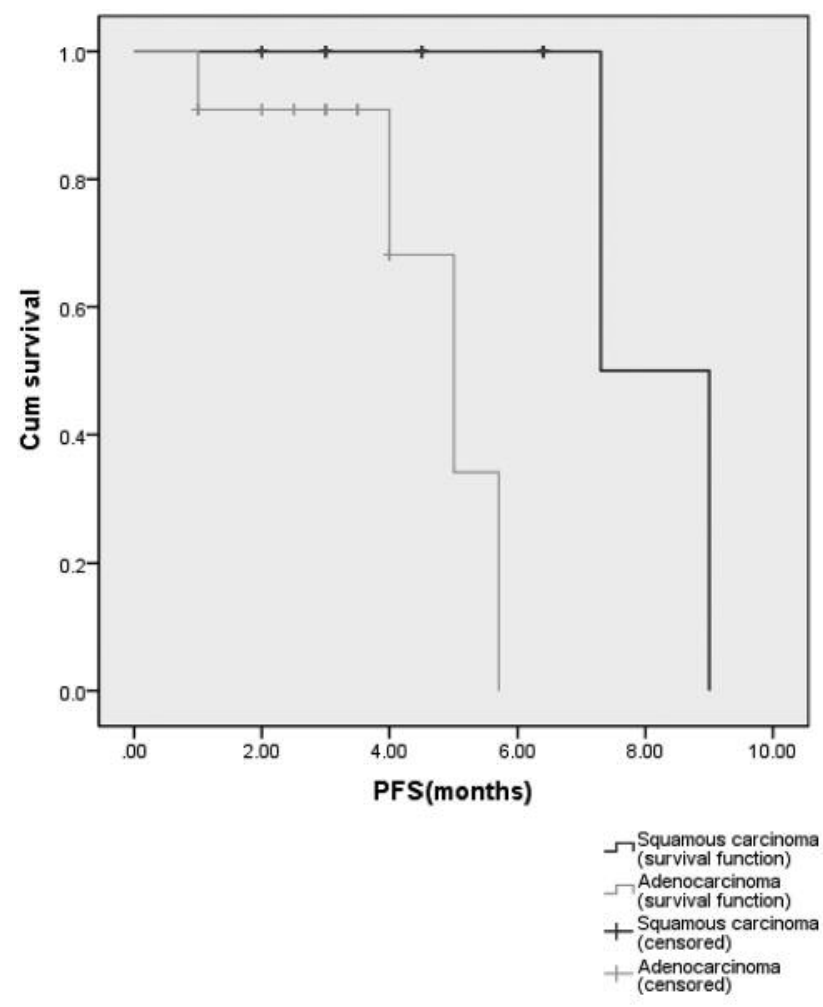

Figure 3. Progression-free survival (PFS) of patients with squamous carcinoma and adenocarcinoma who received nab-paclitaxel as secondline chemotherapy. The median PFS of squamous carcinoma vs. adenocarcinoma was 7.3 vs. 5 months, $p=0.001$.

Standard paclitaxel has a high degree of hydrophobicity and needs poly-oxy-ethylated castor oil as a solvent. The poly-oxyethylated castor oil is commonly associated with hypersensitivity reactions (4-6), peripheral neuropathy, hepatic toxicities and impaired drug delivery (7) for which pretreatment hormone therapy, limited dosage and safety awareness are needed. Some studies have reported that poly-oxy-ethylated castor oil can entrap paclitaxel on solvent micelles making it difficult for the drug to penetrate tumors (6-9).

Nab-paclitaxel is a new paclitaxel and its tolerability, bioavailability and efficacy have been improved compared to standard paclitaxel (10). Nanoparticles of albuminpaclitaxel are absorbed by tumor cells from which paclitaxel is subsequently released to kill tumor cells (11). Compared with standard paclitaxel, nab-paclitaxel has an average particle size of $130 \mathrm{~nm}$ with minimal risk of capillary blockage (12) and enhances delivery of the cytotoxic agent to cancer cells. This occurs via a receptor-mediated transport mechanism as nab-paclitaxel leaves the circulation and enters tissues quicker. As a result, the concentration of nabpaclitaxel is higher in multiple tissues than that of conventional paclitaxel.
Table III. Toxicities of nab-paclitaxel in the patients.

\begin{tabular}{lccccc}
\hline Toxicities & Grade 1 & Grade 2 & Grade 3 & Grade 4 & All \\
\hline Leukopenia & 10 & 5 & 2 & 1 & $52.90 \%$ \\
Thrombocytopenia & 5 & 2 & 1 & 0 & $23.50 \%$ \\
Fatigue & 8 & 0 & 0 & 0 & $23.50 \%$ \\
Nausea/vomiting & 6 & 1 & 0 & 0 & $20.60 \%$ \\
Baldness & 7 & 0 & 0 & 0 & $20.60 \%$ \\
Myalgia & 4 & 2 & 0 & 0 & $17.60 \%$ \\
Peripheral neuropathy & 4 & 1 & 0 & 0 & $14.70 \%$ \\
\hline
\end{tabular}

Nab-paclitaxel was first approved in the United States of America for metastatic breast cancer based on a randomized phase III clinical trial in 2005(13). Nab-paclitaxel combined with carboplatin was approved as first-line treatment of advanced NSCLC by the Food and Drug Administration (FDA) in the United States of America in 2012. It was subsequently approved for the treatment of metastatic pancreatic cancer in 2013. Green et al. (14) proved that nabpaclitaxel was associated with a superior response rate compared with solvent-based paclitaxel on a phase II clinical trial. Nab-paclitaxel plus carboplatin demonstrated a significantly higher efficiency than paclitaxel plus carboplatin as first-line chemotherapy in a phase III clinical trial (3). Further research has also shown that nab-paclitaxel has achieved good results in ovarian, prostate and head and neck cancer (15-17).

In our study, we retrospectively reviewed the treatment outcome of 34 patients with advanced NSCLC who received nab-paclitaxel as second-line chemotherapy. Our study showed that in 34 evaluated patients, ORR was $35.3 \%$ and DCR 70.6\%. Nab-paclitaxel treatment had a high response rate in NSCLC as second-line chemotherapy. Our research found there was no significant difference between clinical features and short-term effect of nab-paclitaxel as secondline chemotherapy. This means nab-paclitaxel can be an appropriate second-line treatment method for patients who have previously been exposed to taxanes or radiotherapy. Socinski et al. (3) reported carboplatin plus nab-paclitaxel had a significantly higher ORR than carboplatin plus solventbased paclitaxel; however, we did not observe this outcome in our study. This difference is potentially due to low patient numbers and/or the use of nab-paclitaxel separately. In our study, there was a significant PFS benefit for patients with squamous lung cancer, compared with patients suffering from lung adenocarcinoma; yet, there was no improvement in OS for squamous lung cancer versus adenocarcinoma. To date, there is no targeted therapy for the treatment of squamous cell lung carcinoma and current treatment options remain limited compared to those for adenocarcinoma. Our data suggest nab-paclitaxel may have a better application 
prospect in this group of NSCLC. In our study, the common adverse events occurred were leukopenia, thrombocytopenia, fatigue, peripheral neuropathy, nausea and vomiting. The most common toxicity was leukopenia that occurred in $52.9 \%$ of patients. After symptomatic and supportive treatment, chemotherapy could continue. Nab-paclitaxel does not need hormone pretreatment and, thus, has minimal effects on blood glucose potentially making it more suitable to minimize the risk of peripheral neuropathy and improve glycemic control. However, this study is small and needs to be continued and expanded enabling further confirmation.

In conclusion, nab-paclitaxel does not cause allergic reactions, increases anticancer activity and reduces toxicity, thus making its administration a viable second-line chemotherapy treatment option in locally advanced and metastatic NSCLC. Kazutoshi et al. (10) hypothesized that secreted protein acidic and rich in cysteine (SPARC), also known as osteonectin, may be a possible predictive marker for selecting patients likely to respond favorably to nabpaclitaxel treatment. Such a theory is, indeed, worthy of extensive investigation.

\section{References}

1 Jemal A, Bray F, Center MM, Ferlay J, Ward E, and Forman D: Global cancer statistics. CA Cancer J Clin 61: 69-90, 2011.

2 Molina JR, Yang P, Cassivi SD, Schild SE and Adjei AA: Nonsmall cell lung cancer: Epidemiology, risk factors, treatment, and survivorship. Mayo Clin Proc 83: 584-594, 2008.

3 Socinski MA, Bondarenko I, Karaseva NA, Makhson AM, Vynnychenko I, Okamoto I, Hon JK, Hirsh V, Bhar P, Zhang H, Iqlesias JL and Renschler MF: Weekly nab-paclitaxel in combination with carboplatin versus solvent-based paclitaxel plus carboplatin as first-line therapy in patients with advanced non-small-cell lung cancer: Final results of a phase III trial. J Clin Oncol 30: 2055-2062, 2012.

4 Gelderblom H, Verweij J, Nooter K, Sparreboom A and Cremophor EL: The drawbacks and advantages of vehicle selection for drug formulation. Eur J Cancer 37: 1590-1598, 2001.

5 Jin F, Zhu H, Shi F, Kong L and Yu J: A retrospective analysis of safety and efficacy of weekly nab-paclitaxel as second-line chemotherapy in elderly patients with advanced squamous nonsmall-cell lung carcinoma. Clin Interv Aging 11: 167-173, 2016.

6 Loong $\mathrm{HH}$, Chan AC and Wong AC: Evolving evidence of the efficacy and safety of nab-paclitaxel in the treatment of cancers with squamous histologies. J Cancer 7: 268-275, 2016

7 Blair HA and Deeks ED: Albumin-bound paclitaxel: A review in non-small cell lung cancer. Drugs 75: 2017-2024, 2015.

8 Van Tellingen O, Huizing MT, Panday VR, Schellens JH, Nooijen WJ and Beijnen JH: Cremophor EL causes (pseudo-) non-linear pharmacokinetics of paclitaxel in patients. $\mathrm{Br} \mathrm{J}$ Cancer 81: 330-335, 1999.
9 Sparreboom A, van Zuylen L, Brouwer E, Loos WJ, De Bruijin P, Gelderblom H, Pillay M, Nooter K, Stoter G and Verweij J: Cremophor EL-mediated alteration of paclitaxel distribution in human blood: Clinical pharmacokinetic implications. Cancer Res 59: 1454-1457, 1999.

10 Hasegawa T, Futamura Y, Horiba A, Yoshida T, Suzuki T, Kato T, Kaito D, Ohno Y, Iida T, Hayashi S and Sawa T: A phase II study of nab-paclitaxel plus carboplatin in combination with thoracic radiation in patients with locally advanced non-smallcell lung cancer. J Radiat Res 57: 50-54, 2016.

11 Kazutoshi Komiya, Tomomi Nakamura, Chiho Nakashima, Koichiiro Takahashi, Hitomi Umeguchi, Naomi Watanbe, Akemi Sato, Yuji Takeda, Shinya Kimura and Naoka Sueoka-Aragane: SPARC is a possible predictive marker for albumin-bound paclitaxel in non-small-cell lung cancer. Onco Targets Ther 9: 6663-6668, 2016

12 Villaruz LC and Socinski MA: Is there a role of nab-paclitaxel in the treatment of advanced non-small cell lung cancer? The data suggest yes. Eur J Cancer 56: 162-171, 2016.

13 Gradishar WJ, Tjulandin S, Davidson N, Shaw H, Desai N, Bhar $\mathrm{P}$, Hawkins $\mathrm{M}$ and O'Shauqhnessy $\mathrm{J}$ : Phase III trial of nanoparticle albumin-bound paclitaxel compared with polyethylated castor oil-based paclitaxel in women with breast cancer. J Clin Oncol 23: 7794-7803, 2005.

14 Green MR, Manikhas GM, Orlov S, Afanasyev B, Makhson AM, Bhar P and Hawkins MJ: Abraxane, a novel CremophoRfree, albumin-bound particle form of paclitaxel for the treatment of advanced non-small-cell lung cancer. Ann Oncol 17: 12631268,2006

15 Teneriello MG, Tseng PC, Crozier M, Encarnacion C, Hancock K, Messing MJ, Boehm KA, Wiliams A and Asmar L: Phase II evaluation of nanoparticle albumin-bound paclitaxel in platinumsensitive patients with recurrent ovarian, peritoneal, or fallopian tube cancer. J Clin Oncol 27: 1426-1431, 2009.

16 Shepard DR, Dreicer R, Garcia J, Elson P, Maqi-Galluzzi C, Raqhavan D, Stephenson AJ and Klein EA: Phase II trial of neoadjuvant nabpaclitaxel in high risk patients with prostate cancer undergoing radical prostatectomy. J Urol 181: 1672-1677, 2009.

17 Damascelli B, Patelli G, Ticha V, Di Tolla G, Frigerio LF, Garbaqnati F, Lancocita R, Marchiano A, Spreafico C, Mattavelli F, Bruno A and Zunino F: Feasibility and efficacy of percutaneous transcatheter intraarterial chemotherapy with paclitaxel in albumin nanoparticles for advanced squamous-cell carcinoma of the oral cavity, oropharynx, and hypopharynx. J Vasc Interv Radiol 18: 1395-1403, 2007.
Received May 19, 2017

Revised June 8, 2017

Accepted June 9, 2017 